\title{
MAG.NETIC TOPOLOGY AND CURRENT SHEET FORMATION
}

\author{
Spiro K. Antiochos \\ E.O. Hulburt Center for Space Research, Naval Research Lab
}

\begin{abstract}
We describe a mechanism for coronal heating. The basic idea is that since the photospheric flux is observed to consist of a complex pattern of positive and negative polarity regions, the topology of the coronal magnetic field (in particular the connectivity) must be discontinuous over a complex network of surfaces and magnetic null points in the corona. Consequently, photospheric motions of the field line footpoints, even if arbitrarily smooth, result in discontinuous stressing of the field. This produces coronal current sheets, reconnection at the null points, and rapid heating.
\end{abstract}

\section{Introduction}

The most widely accepted process for the steady-state heating of the corona is that photospheric motions of coronal field-line footpoints produce currents in the corona. However, for typical coronal temperatures, $\sim 10^{6} \mathrm{~K}$ and size scales, $\sim 10^{8} \mathrm{~cm}$, classical resistive dissipation is too slow to provide the observed power. One approach to circumvent this problem is to decrease the size-scale of the coronal electric currents. Hence, the heating problem reduces in large part to finding an effective mechanism for current filamentation in the corona. In wave heating, resonance absorption is the obvious mechanism for setting up thin, high-current layers (Davila 1987), but in the DC-current models there is a great deal of controversy over the question of current sheets formation.

Three mechanisms have been proposed. They can be distinguished by the requirements they impose on the topology of the initial magnetic field and on the form of the photospheric velocities. We have shown (Antiochos 1987) that the magnetic topology is characterized by the photospheric connectivity, the photospheric footpoint positions of every coronal field line. The connectivity defines a mapping from the positive to the negative polarity regions on the photosphere. We define the field topology to be well-behaved if and only if this mapping is smooth and one-to-one everywhere.

The sheet formation model with the minimum assumptions is due to Parker $(1983,87)$. He argues that smooth magnetic equilibria exist only under highly artificial assumptions on the field topology such as a symmetry in one dimension. In this model current sheets form spontaneously in the corona even if both the topology and the photospheric motions are well-behaved. This is obviously the most favorable circumstances for coronal heating. The difficulty with this model is its basic premise of a lack of equilibrium. To our knowledge there exists no rigorous proof of this hypothesis. Several authors (c.g. Van Ballegooijen 1985, Antiochos 1987) have argued that well-behaved equilibria do exist, but again without a rigorous proof. Perhaps the most convincing arguments come from numerical simulation. A recent 3-d simulation of force-free magnetic equilibria (Micik et al., 1988) shows no evidence for non-equilibrium and appears instead to find well-behaved equilibria. We conclude that the non-equilibrium model requires much more justification before it can be considered as a viable mechanism for coronal heating.

Another current filamentation model has been proposed by Van Ballegooijen (1986). In his model the initial topology is also well-behaved but the photospheric shear develops near discontinuities. The point is that even if the photospheric velocities are smooth, with a large instantaneous characteristice scale (of order the granule size), if these motions 
continue to mix the field-line footpoints, the coronal field will become sheared on finer and finer scales. Van Ballegooijen argues that the scale of this mixing (or shearing) decreases exponentially with time so that eventually the scale of the resulting currents is small enough for resistive dissipation to be effective.

However, Aly (1984) and others have argued that the natural response of the field to continued shearing is to evolve to a completely open field topology. If so then for a given photospheric velocity, there exists a maximum rate of energy input to the magnetic field, and consequently to the coronal heating. This conclusion is supported by numerical calculations. Yang et al (1986) find that for a cylindrically-symmetric sunspot field that undergoes a constant rate of twisting, the energy input rate vanishes initially when the field is potential, and that it vanishes again at large times when the field becomes open. This result argues against Parker's contention (1083) that the rate of coroual heating increases with decreasing resistivity. Parker's statement may be valid for a field in a closed box since then the field stress can build up indefinitely and the power required to maintain a constant footpoint motion may also increase indefinitely; but, for the open geometry of the corona the power vanishes if the magnetic field becomes open. Hence, a severe constraint on Van Ballegooijen's model, and on any current sheet model, is that it must be able to produce localized regions of large magnetic shear while the overall shear remains too small to disrupt the general field structure.

\section{A Model for Current Sheet Formation}

We believe that coronal-current filamentation requires dropping the assumption that the initial field is well-behaved topologically. Note, this does not mean thit the field itself is discontinuous. On the contrary, the initial field is likely to be potential and therefore analytic in the corona; however, the requirement of a continuous connectivity is much stronger than the requirement of a continuous field. Requiring a continuous connectivity is equivalent to requiring that there be only a single magnetic neutral line on the photosphere, so that the photospheric field consists of only one region of positive and one region of negative polarity. The solar field is never that simple.

An example of a potential field with a discontinuous topology is shown in the following Figure. It is due to three "distinct" (i.e. simply connected) polarity regions on the photosphere. This field could be that of a small dipole embedded in a larger background field. For simplicity the small dipole region is shown as being circular, but the flux could as well be concentrated on the photosphere so that the small flux region has a more loop-like appearance. Clearly, this field has a discontinuous connectivity on the photosphere even though the field itself is analytic. The connectivity is discontinuous along the closed curve $S$ formed by the intersection of the photospheric plane with a closed surfuce separating the two flux systems in the corona, the so-called separatrix. The net flux inside $S$ vanishes as does the net flux outside. Note that the conncetivity is also discontinuous at the two points $P_{1}$ and $P_{2}$. Each field line originating on $S$ can be taken to have either $P_{1}$ or $P_{2}$ as the other footpoint; hence, the connectivity is neither smooth nor one-to-one. The explanation for this discontinuity in the connectivity is that a magnetic null point exists at the interface between the two flux systems. This was first pointed out by Sweet (1958), in connection with a model for flares. It is the null point that allows the field to have a discontinuous connectivity and yet be analytic. At the null, the curvature of the field lines becomes singular, so that field lines that are initially arbitrarily close, become finitely separated as they pass "through" the null. 


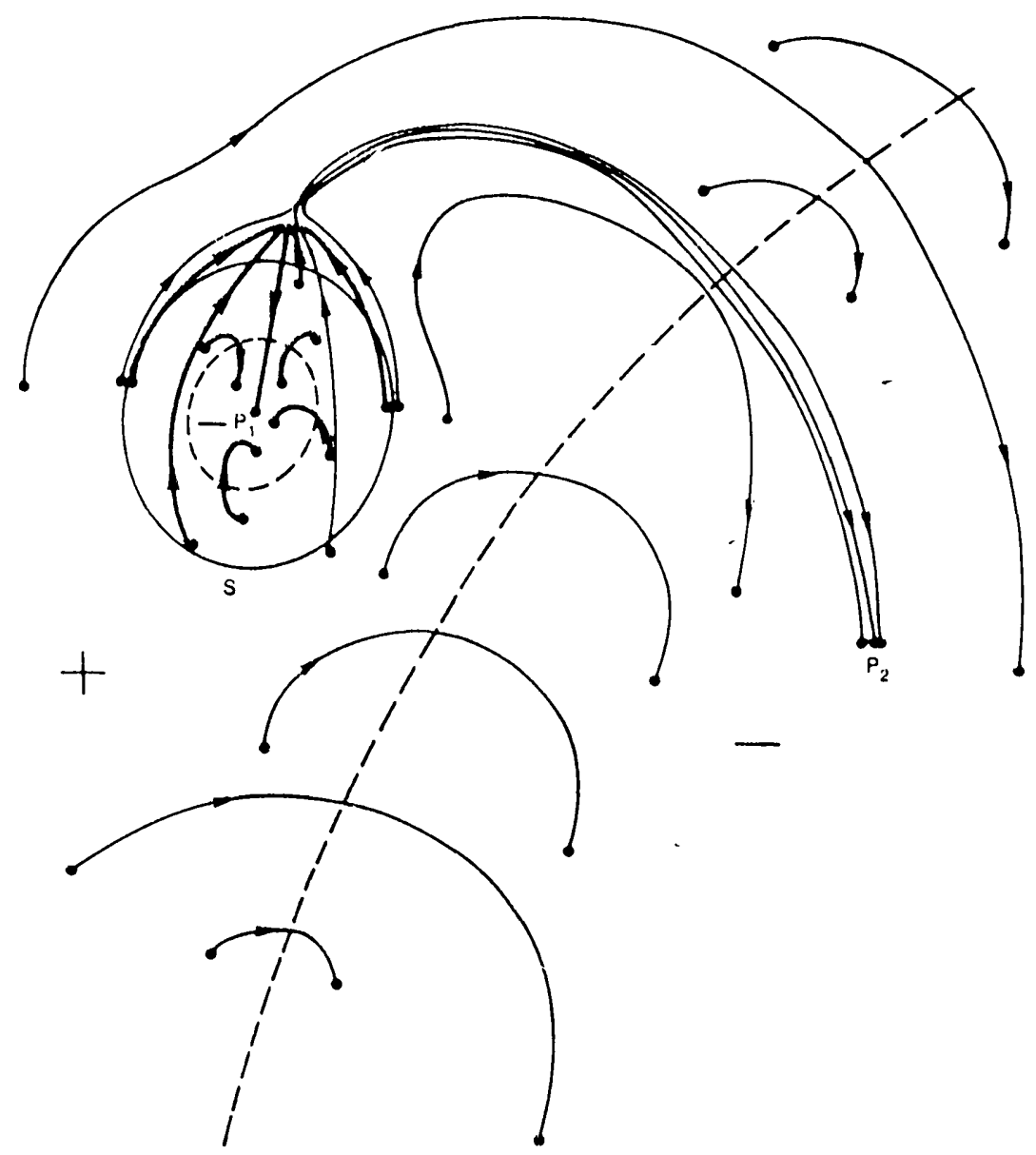

Simple considerations of the model in the Fig. indicate that if the number of distinct polarity regions increases the number of discontinuity lines such as $S$ and the number of null points must increase as well. Observations of the photospheric field indicate that it consists of a complicated mixture of positive and negative polarity regions down to the limit of resolution 2 arcsec (e.g., Dowdy et al., 1986). Strong polarity mixing should be especially prevalent in the network where magnetic flux is convected together by the supergranule flow. In this case there will be a complicated network of separatrix surfaces and lines of discontinuous topology in the corona along with a large number of null points.

Consider the effect of photospheric motions on such a field. The stressing of the field depends on the relative motions of the footpoints, that is on the photospheric velocity and on the connectivity. For a discontinuous connectivity the relative motions will be discontinuous even if the velocity is smooth. Suppose, for example, the photosphere in the Fig. undergoes a small rotational motion $V_{\phi}$ that is centered about the point $P_{1}$ and is localized well within the negative polurity area. This induces a component $B_{\phi}$ only in the small flux system. Since the photosphere is in constant random motion, we expect such magnetic discontinuities, or current filaments, to form along all the separatrixes in the corona, and reconnection to occur at the null points (Syrovatskii 1971). Note that in this model both the assumptions of ideal MHD and of force-free fields are invalid for the solar corona.

We belicve that the null points are a key feature of this model. Greene (1988) has argued that nulls are essential for magnetic reconnection. If so, then rapid energy release may require the presence of magnetic nulis. For example, the tearing mode does not seem to be effective in solar loops unless the field has a true null or, equivalently, field lines closed in the corona (c.g. Velli et al., 1988). Note that unlike null lines or planes, 
which are structurally unstable (Schindler et al., 1988) in that they are destroyed by sinall perturbations of the field, the null point in the Fig. is topologically stable. It survives any footpoint motions as long as the three polarity structure is maintained on the photosplere. Hence, both the assumptions of ideal MHD and of force-free lields are likely to be invalid in regions of complex photospheric sources.

Recently, Low and Wolfson $(1987,88)$ have argued that nulls are not required in order to produce current sheets. They claim that current sheets form wherever the coronal field "dips down" and is tangent to the photosphere along some neutral line. This model is presented as an example of Parker's conjecture, but is actually completely different because the non-equilibrium model assumes an initially well-connected field. The connectivity is discontinuous along the kind of neutral line described by these authors. In fact, their model will not even produce current sheets. The connectivity is discontinuous in their model only if the photosphere is taken to be some infinitely thin, infinitely rigid plane. The photosphere is obviously not such a discontinuity. There is a smooth variation in density and pressure from the corona to the photosphere with gradients there of order the scale height, $\sim 10^{7} \mathrm{~cm}$. If one accounts properly for the response of the photospheric plasma to magnetic stresses, then one concludes that the model by Low and Wolfson will produce only distributed currents with scales of order the scale height (Antiochos 1988). The key difference between their model and ours is that their separatrix surface is determined by the position of the photosphere. Assuming a higher or lower position for the photosphere results in a diflerent separatrix. This is not the case for the field in the Fig. The separatrix is determined as the surface formed by all the field lines that pass through the mull point. This is a true topological feature of the field, and is independant of the assumed photospheric position.

In summary, we conclude that null points and the type of structure shown in the Fig. may play a major role in coronal heating. The heating rates predicted by this model will be discussed in a future paper.

\section{References}

Aly, J.J. 1984, Ap. J., 283, 349.

Antiochos, S.K. 1987, Ap. J., 312, 886.

Antiochos, S.K. 1988, in preparation.

Davila, J.M. 1987, Ap. J., 317, 514.

Dowdy, J.F., Jr., Rabin, D. and Moore, R.L. 1986, Solar Phys., 105, 35.

Greene, J.M. 1988, J. Geophys. Res., 93, 8583.

Low, B.C. 1987, Ap. J., 323, 358.

Low, B.C. and Wolfson, R. 1988, Ap. J., 324, 574.

Mikic, Z., Schnack, D.D. and Van Hoven, G. 1988, Ap. J., submitted.

Parker, E.N. 1983, Ap. J., 264, 642.

Parker, E.N. 1987, Ap. J., 318, 876.

Schindler, K., Hesse, J. and Birn, J. 1988, J. Geophys. Res., 93, 5547.

Sweet, P.A. 1958, Nuovo Cimento Suppl., Ser. X 8, 188.

Syrovatskii, S.I. 1971, Soviet Phys. JETP 33, 933.

Van Ballegooijen, A.A. 1985, Ap. J., 298, 421.

Van Ballegooijen, A.A. 1986, Ap. J., 311, 1001.

Velli, M., Einaudi, G. and Hood, A.W. 1988, this volume.

Yang, W.H., Sturrock, P.A., and Antiochos, S.K. 1986, Ap. J., 309, 383. 